\title{
Open Source Software for Model Driven Development: A Case Study
}

\author{
Jonas Gamalielsson $^{1}$, Björn Lundell ${ }^{1}$, and Anders Mattsson ${ }^{2}$ \\ ${ }^{1}$ University of Skövde, Skövde, Sweden \\ \{jonas.gamalielsson, bjorn. Iundell\} @his.se \\ ${ }^{2}$ Combitech AB, Jönköping, Sweden \\ anders.mattsson@combitech.se
}

\begin{abstract}
Model Driven Development (MDD) is widely used in the embedded systems domain, and many proprietary and Open Source tools exist that support MDD. The potential for sustainability of such tools needs to assessed prior to any organisational adoption. In this paper we report from a case study conducted in a consultancy company context aiming to investigate Open Source tools for MDD. For the company it was interesting to explore the two Open Source modelling tools Topcased and Papyrus for potential adoption. The focus for our case study is on assessing the health of the ecosystems for the two investigated Open Source projects by means of quantitative analysis of publically available data sources about Open Source projects. The health of ecosystems is an important prerequisite for a long term sustainable OSS (Open Source Software) tool-chain in the MDD area, which can aid strategic decision making for potential adoption within a company context. We have established details on the extent to which developers and users are active in two specific OSS ecosystems, and identified organisational influence for both ecosystems. We find that the investigated tools are promising regarding the health of their ecosystems, and a natural next step for the company would be to proceed with a pilot study in order to analyse the effectiveness of the investigated tools in company contexts.
\end{abstract}

\section{Introduction}

Despite the existence of many tools supporting Model Driven Development (MDD), there is currently a lack of reported case studies from MDD practice in industry (Mattsson et al. 2009). Some of these tools are licensed as Open Source Software (OSS). There are a number of different motivations for utilising OSS within companies, and companies have adopted several different business models for engaging in Open Source (Bonaccorsi and Rossi 2006; Lundell et al. 2010; van der Linden et al. 2009). In fact, the majority of today's innovative products and solutions are developed on the basis of Open Source software (Ebert 2008). In recent years, there has been an increased interest in OSS usage amongst practitioners in Swedish companies, with an increased emphasis on active involvement in OSS projects beyond passive use of OSS products (Lundell et al. 2010). In this paper we report from an analysis of two MDD tools (Topcased (Topcased.org 2011a) and Papyrus 
(Eclipse.org 2011a)) provided as Open Source software, with the goal to aid strategic decision making for potential adoption within a company context.

Some of the contributors in the OSS projects are affiliated with companies that provide support and consultant services related to the OSS projects at hand. On the other hand, other contributors are affiliated with companies in the secondary software sector (Fitzgerald 2006), i.e. companies with extensive software development in domains such as avionics, automotive, telco, etc., but whose main goal is not to provide services to OSS projects. For companies in such domains, developed solutions often require long term maintenance, something which developers in this area see "as fundamentally important" (Lundell et al. 2011). For example, support for products in the avionics domain will often last for more than 70 years (Robert 2007). Further, previous results from the embedded systems area show that support from large OSS communities is "considered superior compared to proprietary alternatives in some cases." (Lundell et al. 2011)

In recent years we have seen a commodification in software development (van der Linden et al. 2009), and many companies in the secondary software sector develop and are dependent upon a large amount of software which do not give a competitive advantage. For such commodity software, many large and small companies seek to leverage from the Open Source licensing model in their collaboration on commodity software. An example of such commodity software is the Eclipse platform, which constitutes the basis for many Open Source licensed development tools (West 2003).

Before an organisation adopts an Open Source project it is important to evaluate its ecosystem in order to make sure that it is healthy and that the project is likely to be sustainable and maintained for a long time (van der Linden et al. 2009). One important means in such an evaluation is to quantitatively assess the health of an Open Source community (Crowston and Howison 2006). A number of studies have investigated large, well known Open Source projects through quantitative analysis, including the Linux kernel (Moon and Sproull 2000), Apache (Mockus et al. 2002), Mozilla (Mockus et al. 2002), Gnome (German 2004) and KDE (Lopez-Fernandez et al. 2006). Such quantitative assessment includes, but is not limited to, analysis of data from: Software Configuration Management (SCM) systems such as CVS and SVN (Lopez-Fernandez et al. 2006; Gamalielsson and Lundell 2010), mailing lists (Kamei et al. 2008; Gamalielsson et al. 2010), and bug tracking systems (Crowston and Howison 2005; Mockus et al. 2002).

Our study is undertaken as a case study conducted in a medium sized consultancy company active in the embedded systems domain. The goal has been to investigate the two Eclipse-based Open Source projects Topcased and Papyrus for potential company adoption. Specifically, the aim is to reveal insights concerning the health of the ecosystems of the two OSS projects as this constitutes an important basis for strategic decision making within the company.

\section{Research Approach}

In this paper we report from a case study conducted within the consultancy company Combitech AB (hereafter referred to as Combitech), which is a company working with advanced systems design and software development, electronic engineering, process optimisation, and staff training. It has approximately 800 employees and 
covers a broad spectrum of business areas such as defence, aviation, automotive, medical and telecoms.

The company has a long experience of systematic method work and model-based system development. In several development projects, UML is used (e.g., Mattsson et al. (2009)), but other modelling techniques are used as well. The company has experience from use of three major case tools supporting both UML and time-discrete modelling: Rose Realtime ${ }^{\circledR}$ (from IBM), Rhapsody® (from IBM and previously iLogix), and TAU® (from Telelogic). Combitech has an interest in exploring the potential of the Eclipse platform and Open Source tools to complement (and possibly replace) its current tool suite.

For Combitech it was interesting to explore the two Open Source modelling tools Topcased (Topcased.org 2011a) and Papyrus (Eclipse.org 2011a) for potential adoption. A motivation for conducting a study involving Papyrus and Topcased is that both tools are based on the Eclipse Modelling Framework (EMF). With an organisational adoption of EMF, it will be possible to exchange models between the many tools supporting EMF, thus minimising the risk for lock-in and enabling integration of tools to support company development practices with effective tool chains. Further, initial use of Topcased within the company context was a positive experience, which increased the interest in further exploration.

Topcased is a software environment primarily intended for development of critical embedded systems encompassing both software and hardware. It promotes the use of model-driven engineering and formal methods. Topcased stems from an industrial R\&D project (Lundell et al. 2008), and is released as Open Source by a partner group where members originate from various organisations. According to Eclipse.org (2010), Topcased "is backed by some major industrial players". At time of writing (January 2011), the project contains approximately 8000 source code files. $71 \%$ of these are Java files, in total containing 1.2 MLOC (excluding comments and blank rows) (Ohloh.net 2011a).

Papyrus is a graphical editing tool for models in languages such as UML, SysML and MARTE. Papyrus was included in the MDT (Model Development Tools) subproject of the Eclipse platform in January 2009. The project currently contains approximately 9400 source code files, where $73 \%$ of these are Java files which in total contain 2.3 MLOC (Ohloh.net 2011b).

To guide the decision on whether to engage in any of the ecosystems for the two projects, Combitech wanted to establish that the projects are likely to be sustainable and maintained for a long time. This was considered important since any organisational adoption of an OSS MDD tool-chain implies a long-term commitment which affects working practice within the company. Specifically, three aspects of ecosystem health were considered important: activity in ecosystems; company influence in ecosystems; and interaction between ecosystems for the two OSS projects (Topcased and Papyrus). The company interest in these three aspects of ecosystem health is in line with previous research (Crowston and Howison 2006; German 2004; Gamalielsson et al. 2010).

To investigate the activity in ecosystems, we analysed the contributions in terms of committed SCM artefacts of the Open Source projects over time (from start of projects to August 2010). To investigate the company influence in ecosystems, we analysed over time the extent to which different contributors are affiliated with different companies. The interaction between ecosystems was assessed by studying 
contributors that are active in both projects. The data for Topcased was collected from the Gforge website for the project (http://gforge.enseeiht.fr/projects/topcased, accessed 10 September 2010), and for Papyrus the data was collected from the Eclipse website for the project (http://www.eclipse.org/projects/project_summary .php?projectid=modeling.mdt.papyrus, accessed 10 September 2010).

\section{Results}

This section presents results on the activity in the two OSS projects Topcased and Papyrus, an analysis of how different contributors are affiliated with different companies, and the interaction between the two ecosystems.

\subsection{Activity in Ecosystems}

The activity in ecosystems is an important factor that reflects the health and long term sustainability of OSS projects, and we therefore studied each project separately in terms of the extent of contributions to SCM repositories, mailing lists, and forums over time.

The number of commits for Topcased as a function of time is shown in Figure 1. The first commits were made in November 2004 and the last month studied is August 2010 (70 months in total). It can be observed that a major part of the commits are code commits (red trace), constituting on average $60 \%$ of the total number of commits

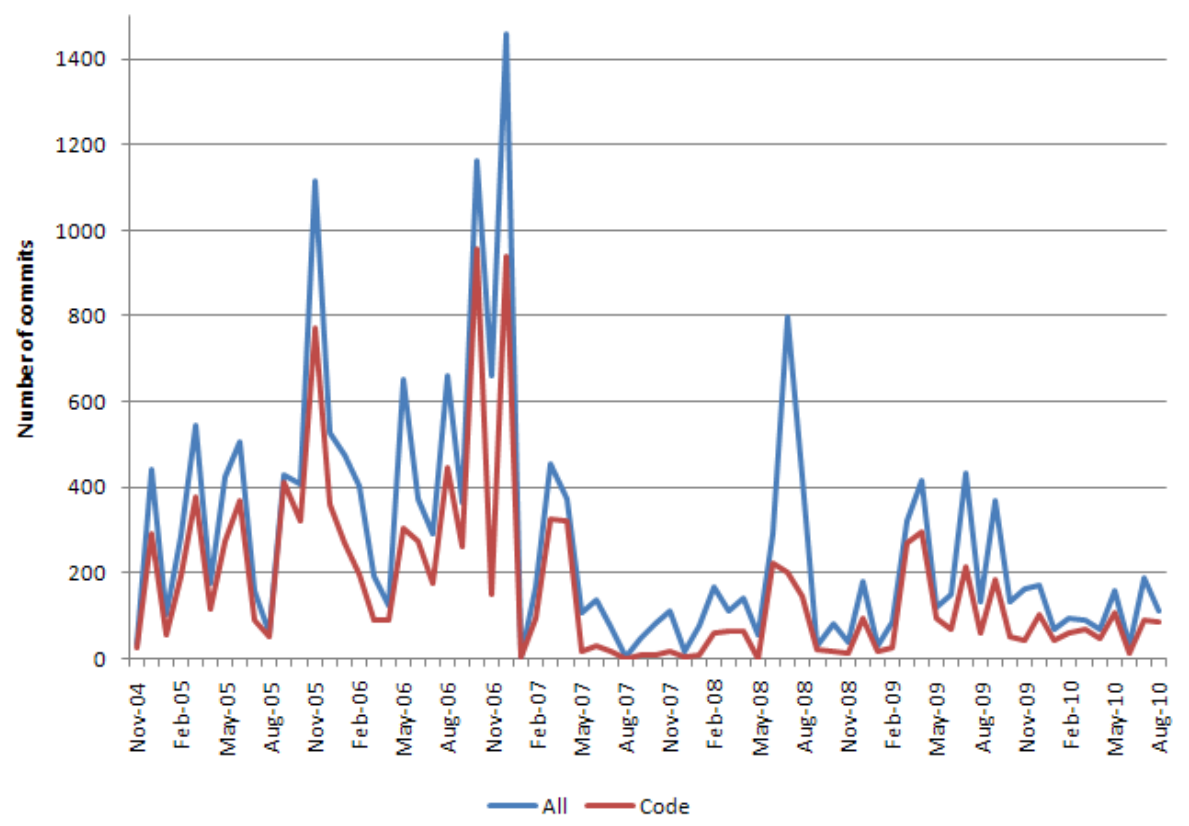

Fig. 1. Topcased: number of commits 
(blue trace). Another fact is that some peaks (e.g. November 2005, November 2006 and July 2008) co-occur with events in the project version history shown in Table 1 . It can be noted that there has been a large number of releases since the start of the project.

The number of messages contributed to the developer- and user mailing lists of Topcased is illustrated in Figure 2. Like in Figure 1, the peaks can be related to the version history in Table 1. It can be noted that there is elevated activity in the developer list around May 2005 (before the initial release) and also at the time of the initial release (October 2005) and version 2.0.0 (July 2008). Similarly, peaks in the user mailing list often co-occur with events in the version history (e.g. September 2006, May 2007 and May 2009). Some peaks in Figure 2 also co-occur with peaks in Figure 1 (e.g. October 2006, July 2008 and April/May 2009). The average number of postings each month is 21 for the developer mailing list and 67 for the user mailing list, which shows that there is more activity on average in the user mailing list.

The number of commits for Papyrus as a function of time is shown in Figure 3. SCM data for this project spans from January 2009 to August 2010 (20 months in total). It can be noted that code artefacts are dominating (79\% of total number of commits). The commit activity is high during the entire period of study, and the three largest peaks occur in February 2009, December 2009 and July 2010. The first two of these peaks do not co-occur with events in the version history of Papyrus (see table 2 ), but the last peak co-occurs with the release of version 0.7 . So far, there has only been a few releases of the tool.

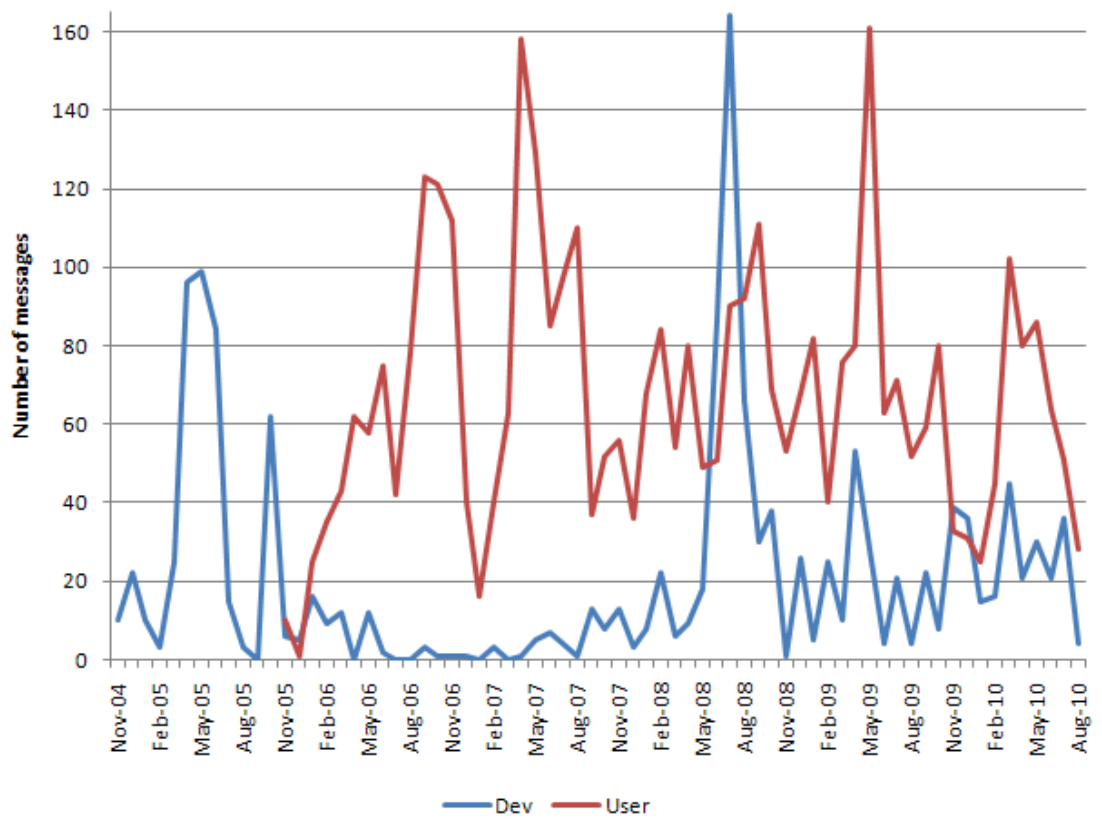

Fig. 2. Topcased: number of messages in developer- and user mailing lists 
Table 1. Topcased: version history

\begin{tabular}{|c|c|}
\hline Date (Y-M-D) & Release \\
\hline $2005-10-11$ & Initial \\
\hline 2005-11-17 & 0.6 .0 \\
\hline 2006-01-24 & 0.7 .0 \\
\hline 2006-03-17 & 0.8 .0 \\
\hline 2006-06-07 & 0.9 .0 \\
\hline $2006-09-20$ & 0.10 .0 \\
\hline 2006-11-08 & 0.11 .0 \\
\hline $2007-05-29$ & 1.0.0M4.1 \\
\hline 2007-08-11 & 1.0 .0 \\
\hline $2007-11-26$ & $2.0 .0 \mathrm{M} 3$ \\
\hline 2007-12-07 & 1.2 .0 \\
\hline $2008-01-20$ & $2.0 .0 \mathrm{M} 4$ \\
\hline $2008-02-20$ & 1.3 .0 \\
\hline $2008-03-03$ & $2.0 .0 \mathrm{M} 5$ \\
\hline 2008-04-19 & $2.0 .0 \mathrm{M} 6$ \\
\hline $2008-07-18$ & 2.0 .0 \\
\hline 2008-09-01 & 2.1 .0 \\
\hline 2008-11-06 & 2.2 .0 \\
\hline 2009-01-07 & 2.3 .0 \\
\hline 2009-03-16 & 2.4 .0 \\
\hline 2009-05-07 & 2.5 .0 \\
\hline $2009-07-27$ & $3.0 .0 \& 2.6 .0$ \\
\hline $2009-09-28$ & 3.1 .0 \\
\hline 2009-12-03 & 3.2 .0 \\
\hline $2010-03-22$ & 3.3 .0 \\
\hline 2010-06-02 & 3.4 .0 \\
\hline $2010-06-21$ & 3.4 .1 \\
\hline $2010-07-23$ & 4.0 .0 \\
\hline
\end{tabular}

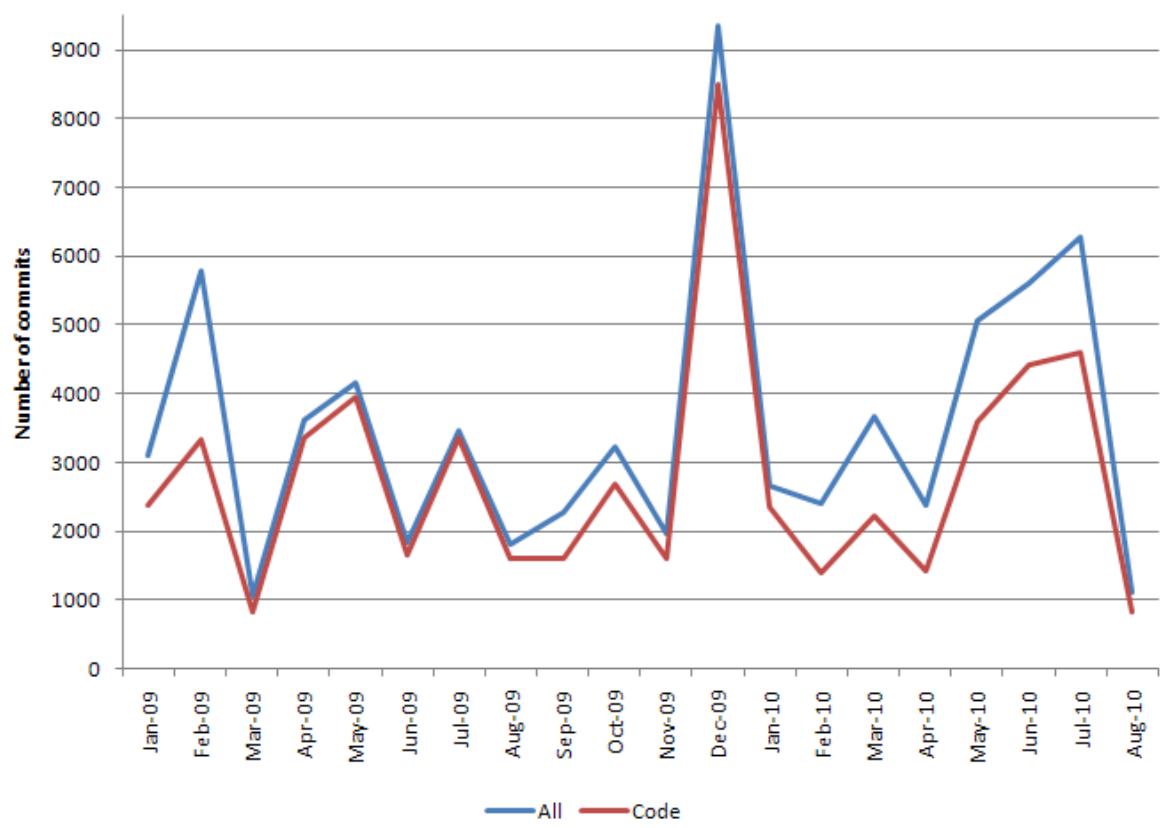

Fig. 3. Papyrus: number of commits 
The number of messages contributed to the developer mailing list and forum of Papyrus is illustrated in Figure 4. It can be observed that the two largest peaks for the developer mailing list co-occur with the initial- (November 2008) and second milestone release (October 2009) of Papyrus. The peaks for the forum are less distinct. The average number of postings each month is 47 for the developer mailing list and 27 for the forum, which shows that there is more activity on average in the developer mailing list.

Table 2. Papyrus: version history

\begin{tabular}{|c|c|}
\hline Date (Y-M-D) & Release \\
\hline $2008-11-25$ & Initial \\
\hline $2009-09-18$ & $0.7 \mathrm{M} 1$ \\
\hline $2009-10-16$ & $0.7 \mathrm{M} 2$ \\
\hline $2010-07-14$ & 0.7 \\
\hline
\end{tabular}

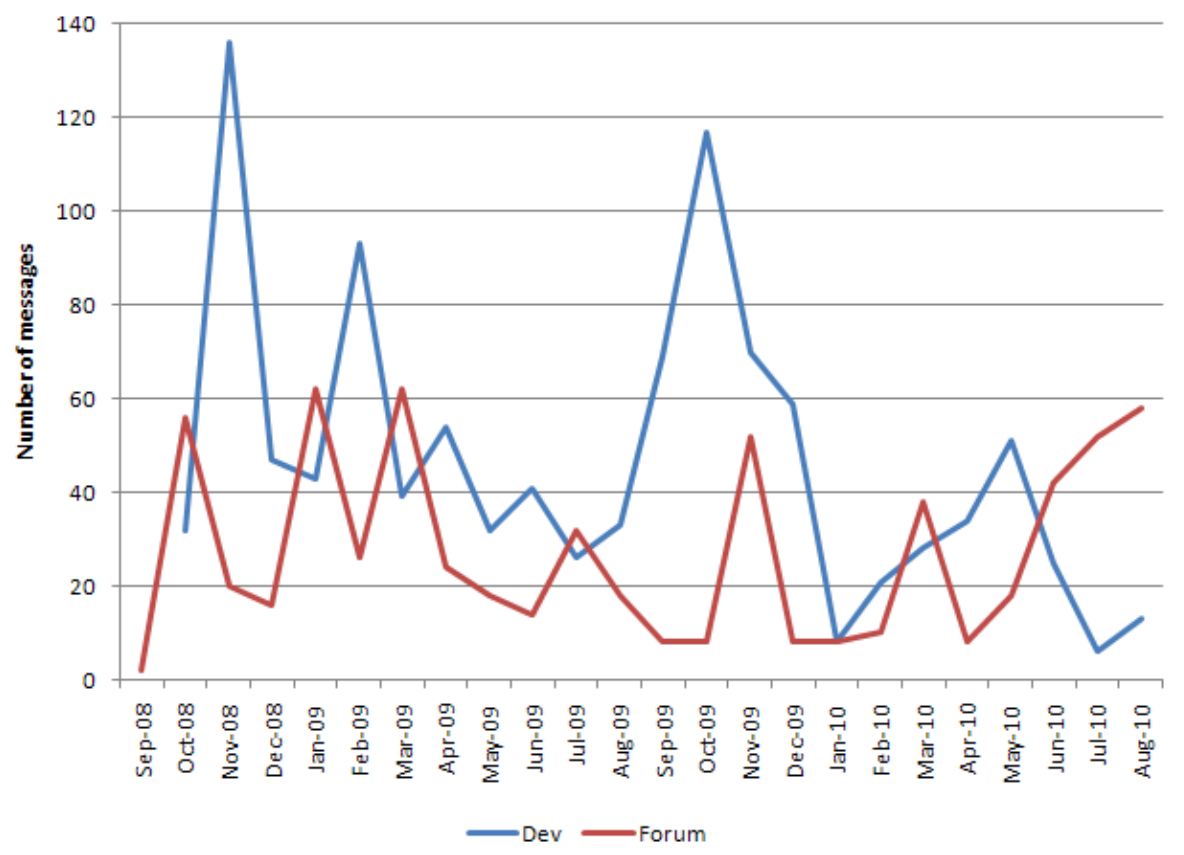

Fig. 4. Papyrus: number of messages in developer- and user mailing lists

\subsection{Influence in Ecosystems}

It was previously known to Combitech that company influence over time can affect the long term sustainability of a project, and we therefore studied each project separately in terms of contribution and affiliation.

Figure 5 illustrates the proportion of SCM commits for the eight different affiliations for Topcased. It can be observed that different affiliations are dominating 
in different time periods. Affiliations are also ranked from most influential over all time (A1) to least influential (A8). Affiliation A1 (blue) is dominating from November 2004 until August 2008, whereas A2 (red) and A3 (green) together largely dominate from September 2008 until August 2010. The remaining affiliations (A4 through A8) are considerably less influential. The orange colour represents unknown affiliations.

Figure 6 shows the corresponding proportion of SCM commits for the six different affiliations for Papyrus. Like for Topcased, affiliations are ranked from most influential over all time (A9) to least influential (A12). It can be noted that A9 is the most influential affiliation over all time, and is dominating during most months. A2 (which was also active in Topcased) is active from April 2009 until August 2010, and is dominating during some months. A4 (also active in Topcased) is the third most influential affiliation, and is dominating in April and May 2009.

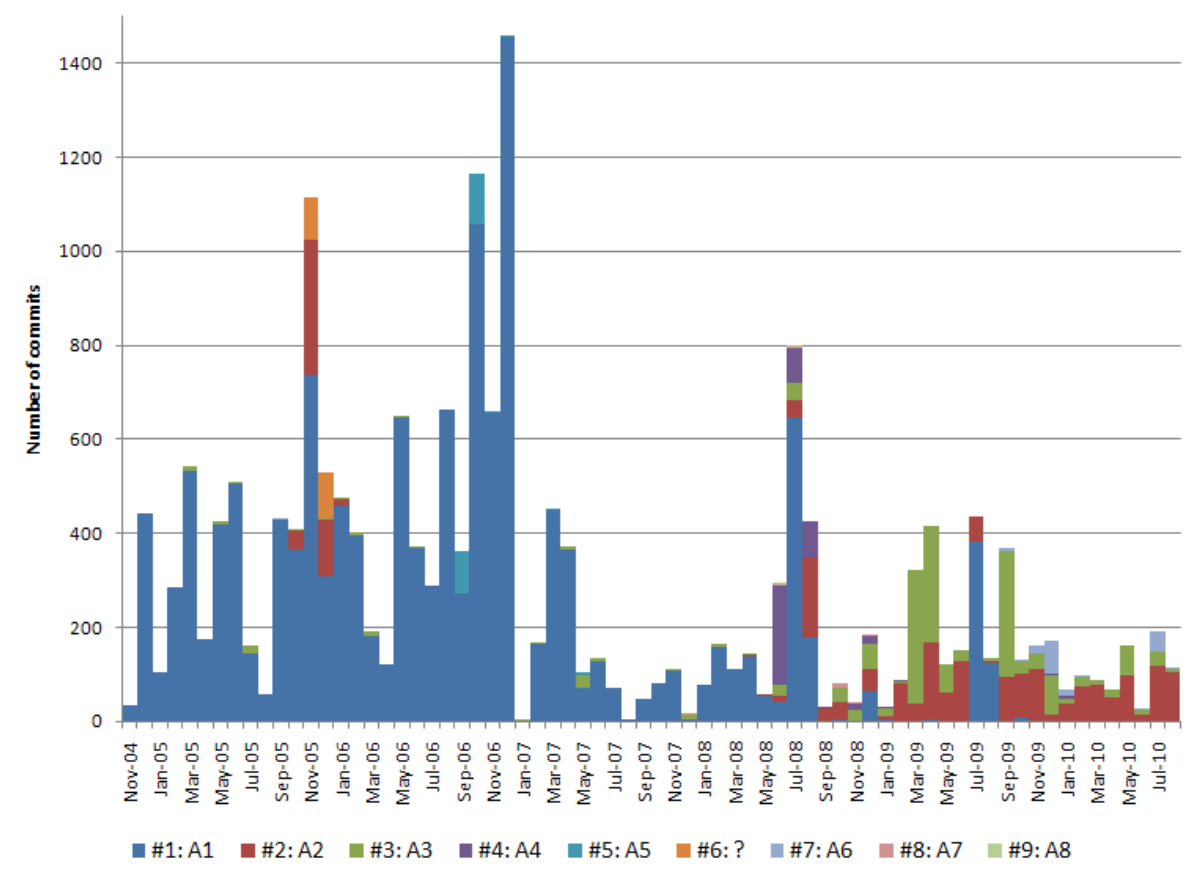

Fig. 5. Topcased: Proportion of commits per affiliation over time

Figure 7 illustrates the total proportion of SCM contributions for different affiliations in Topcased (left pie chart) and Papyrus (right pie chart). It is evident that for both projects, one single affiliation is clearly dominating (A1 for Topcased with $76 \%$ of the commits, and A9 for Papyrus with $68 \%$ of the commits). For both Topcased and Papyrus, the three most influential affiliations account for approximately $95 \%$ of the commits. 


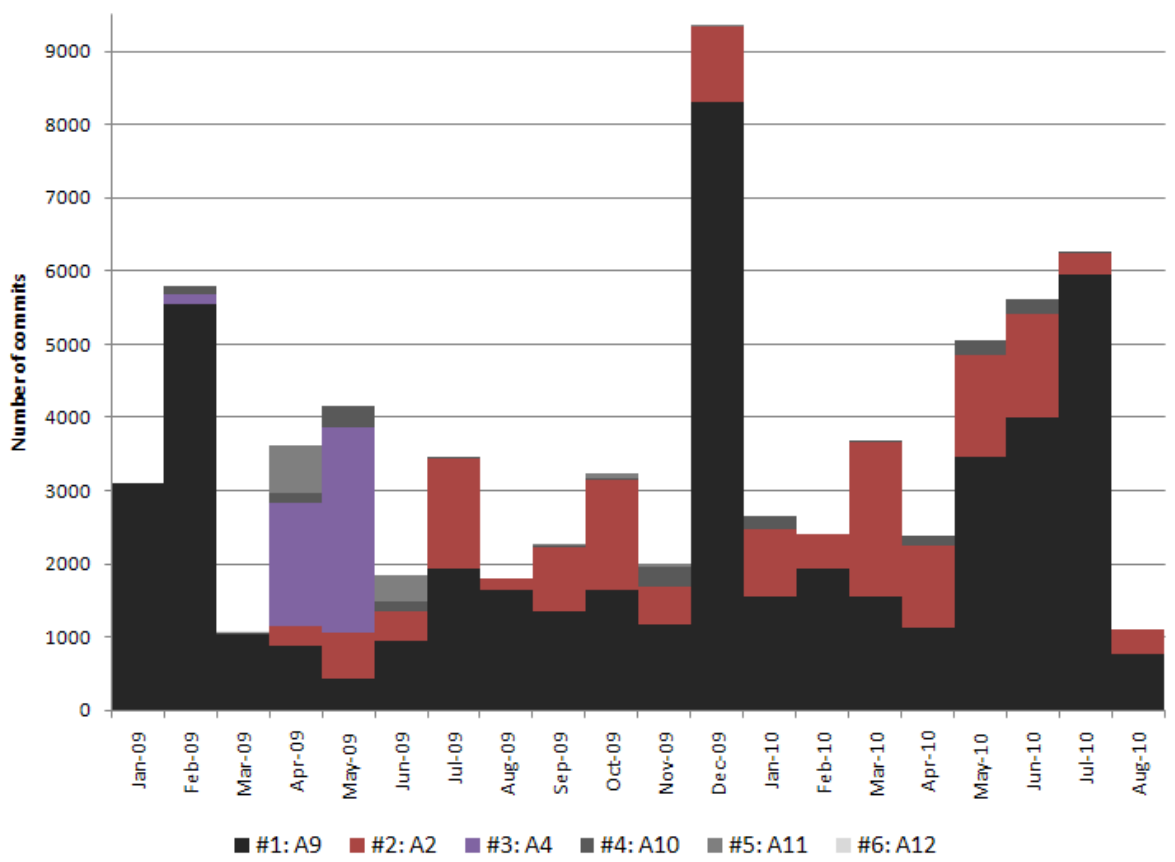

Fig. 6. Papyrus: Proportion of commits per affiliation over time
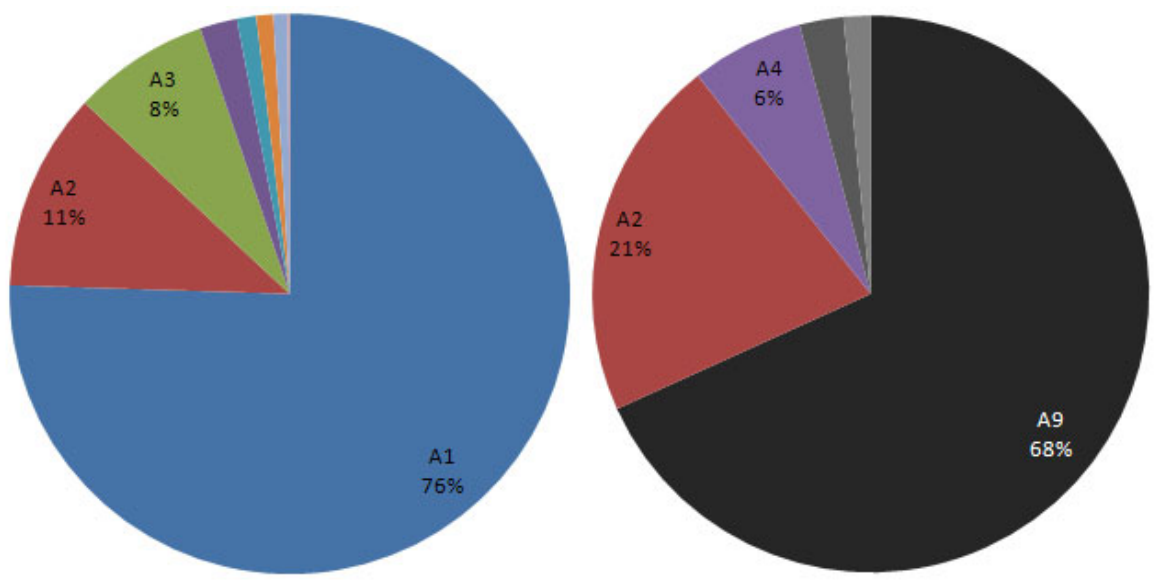

Fig. 7. Total affiliation commit influence (left pie: Topcased, right pie: Papyrus)

The affiliations can be classified into different affiliation types: Small and Medium Enterprise (SME), Large Company (LC) and Research Institute (RI), see Table 3. 
Table 3. Affiliation type (rows) for affiliations (columns)

\begin{tabular}{|c|c|c|c|c|c|c|c|c|c|c|c|c|}
\hline & A1 & A2 & A3 & A4 & A5 & A6 & A7 & A8 & A9 & A10 & A11 & A12 \\
\hline SME & X & & & X & & & X & X & & & X & X \\
\hline LC & & X & X & & X & X & & & & & & \\
\hline RI & & & & & & & & & $X$ & $X$ & & \\
\hline
\end{tabular}

The proportion of SCM commits for different affiliation types is illustrated in Figure 8 for Topcased. It can be observed that SME (blue) is dominating from November 2004 until August 2008, whereas "large company" (red) largely dominates from September 2008 until August 2010.

Figure 9 shows the corresponding proportions of SCM commits for Papyrus. It is evident that the most influential affiliation is "research institute" (brown), which is active during all months and dominating during the majority of the months. SME (blue) is most active, and dominating, in April and May 2009. "Large company" (red) is active from April 2009 until August 2010, and is occasionally dominating.

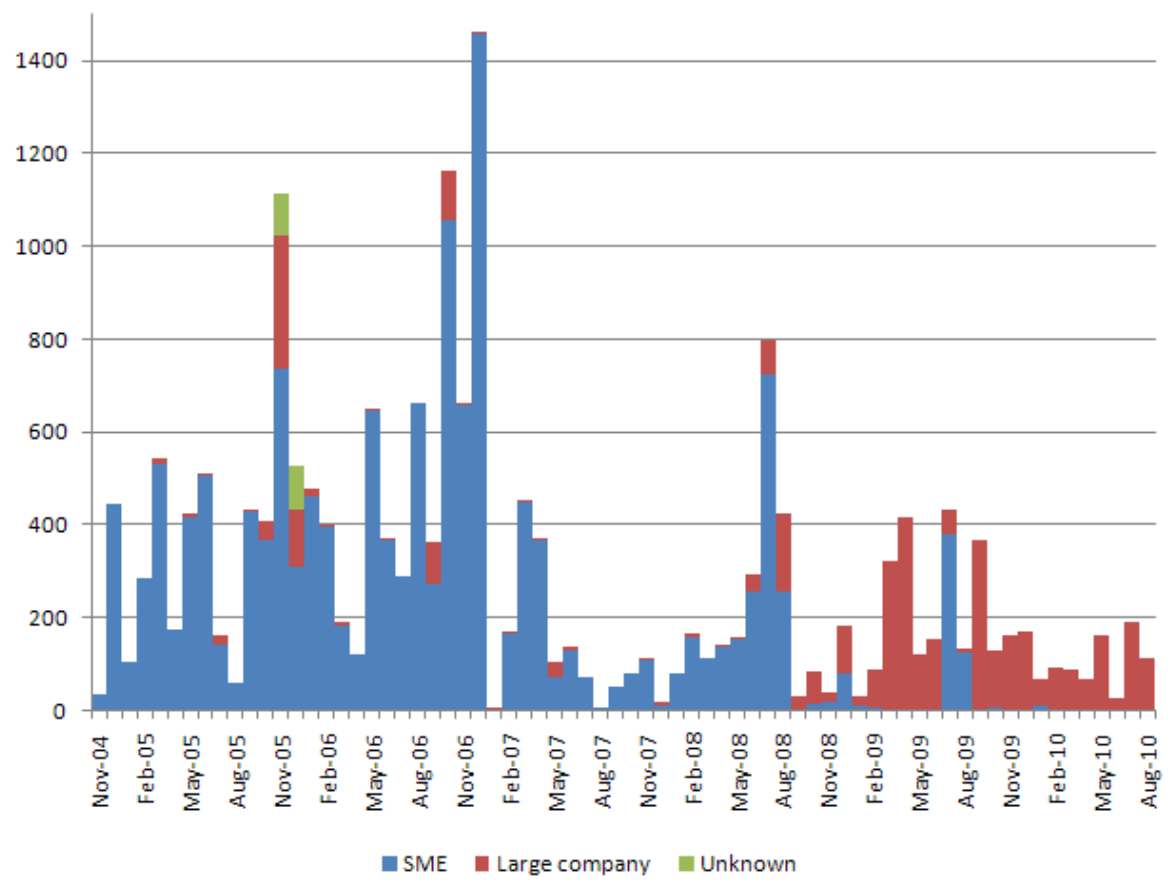

Fig. 8. Topcased: number of commits for different affiliation types

Figure 10 illustrates the total proportion of SCM contributions for different affiliation types in Topcased (left pie chart) and Papyrus (right pie chart). It is evident that for both projects, one single affiliation type is dominating (SME for Topcased with $78 \%$ of the commits, and "research institute" for Papyrus with $71 \%$ of the commits). It is interesting to note that "large company" has the same proportion of commits $(21 \%)$ for both projects. 


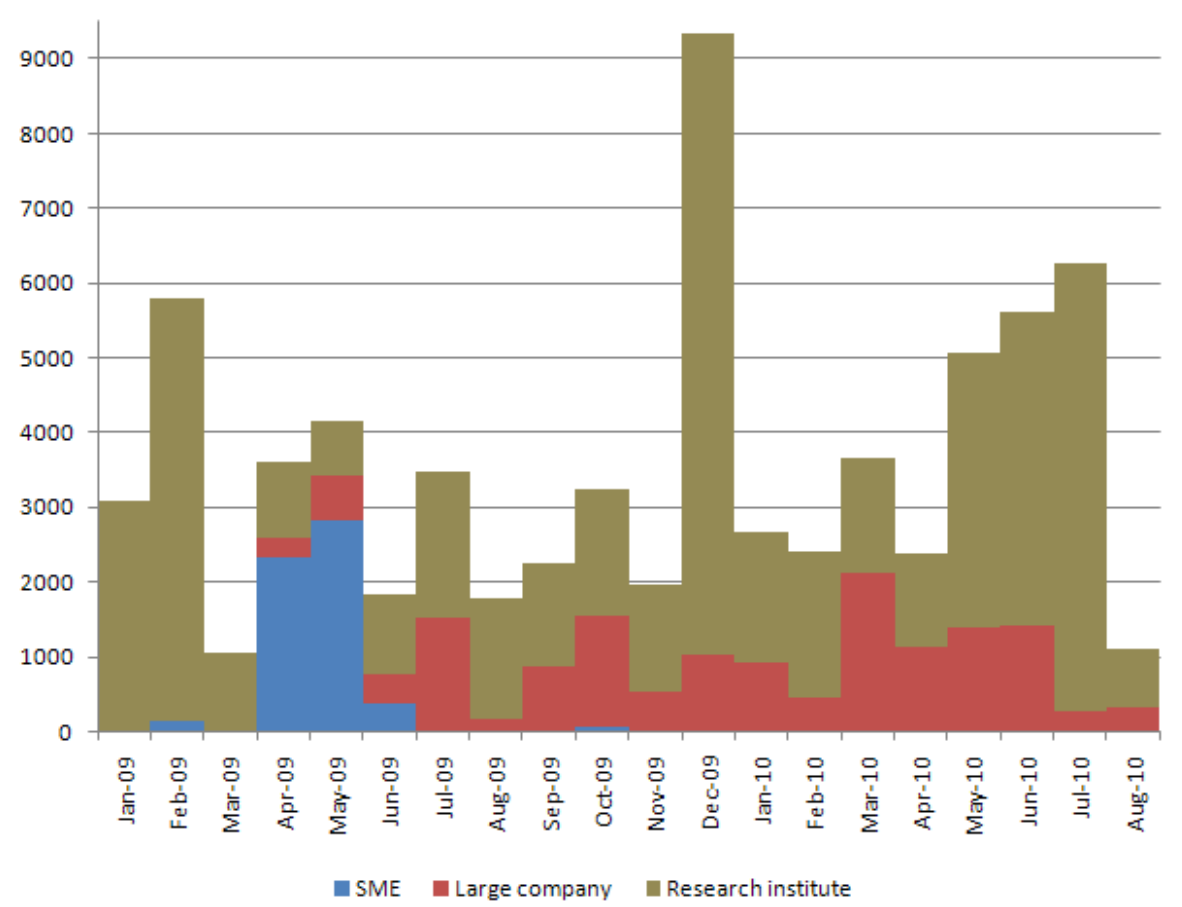

Fig. 9. Papyrus: number of commits for different affiliation types
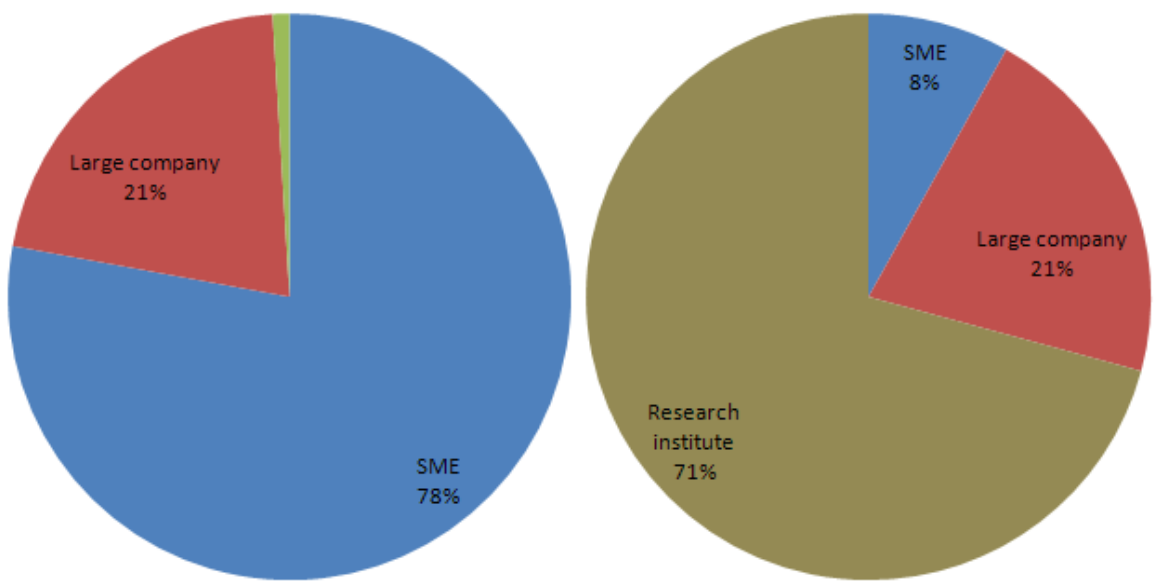

Fig. 10. Total affiliation type commit influence (left pie: Topcased, right pie: Papyrus)

The influence can also be studied at committer level. The number of SCM commits from the top committers (those who have made largest proportion of commits over all time) in Topcased as a function of time, is shown in Figure 11. It can be observed that the single most influential committer (blue trace) is dominating in several periods and 


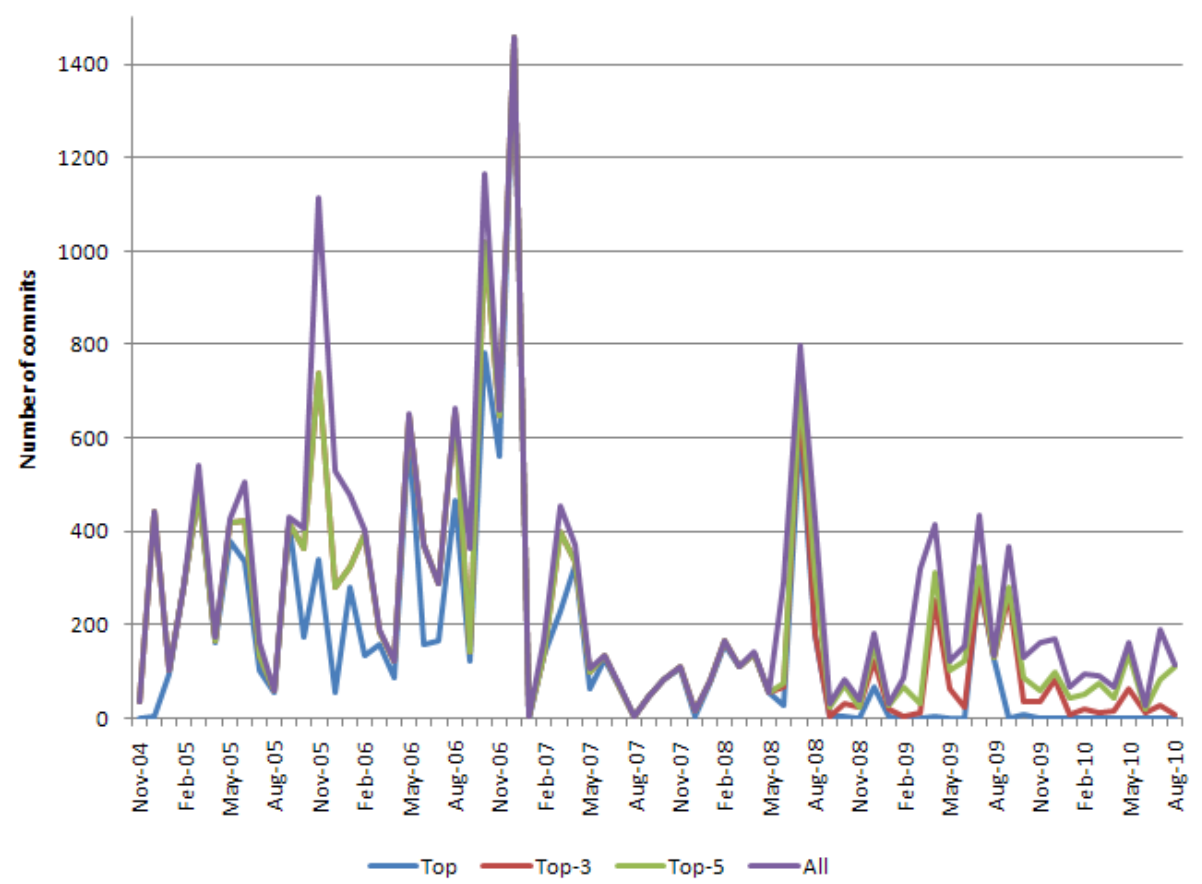

Fig. 11. Topcased: number of commits for top committers

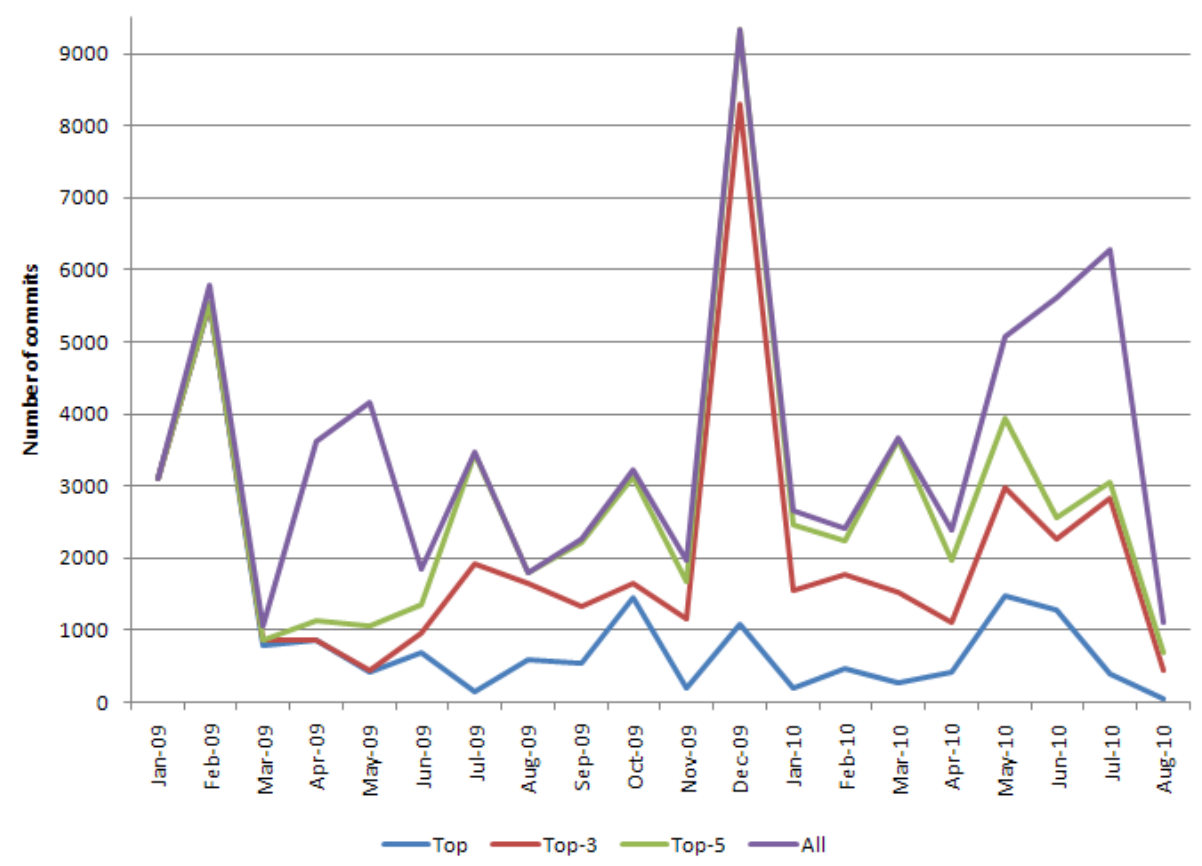

Fig. 12. Papyrus: number of commits for top committers 
contributes $56 \%$ of all commits. The top three committers (red trace) and top five committers (green trace) largely follow the trace of all commits (purple colour), and contribute $78 \%$ and $84 \%$ of all commits, respectively.

The number of SCM commits from the top developers in Papyrus is illustrated in Figure 12. It can be noted that the single most influential committer (blue trace) is dominating during the first three months, but is thereafter less influential, and contributes $28 \%$ of all commits. Further, there is a bigger difference between the contribution from the top three and top five committers, who contribute $60 \%$ and $78 \%$ of all commits, respectively.

\subsection{Interaction between Ecosystems}

For Combitech it was interesting to investigate the extent to which individual developers contribute to both OSS projects over time. Such insights may indicate future relevance of the projects for Combitech since both projects are likely to benefit from exchange of expertise, which in turn can increase innovation in the projects.

Figures 13 and 14 show the contribution of the inter-project committers for Topcased and Papyrus during the life span of both projects. For example, Figure 13 shows that $38 \%$ of all (160 of totally 416) commits to Topcased in April 2009 stem from developers active in both projects. There are four committers that are active in

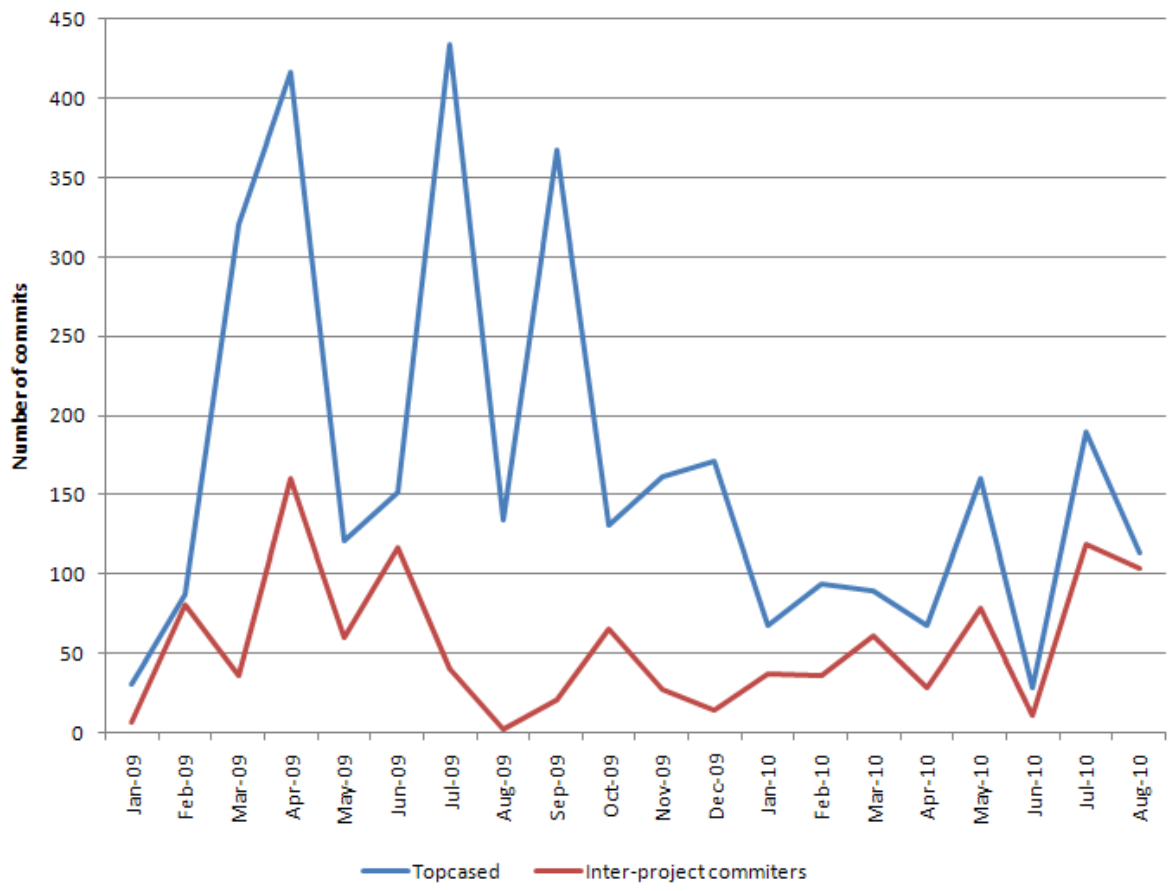

Fig. 13. Topcased: total number of commits and contribution by inter-project committers (during the life span of Papyrus) 
both projects during the life span of the projects. These committers are all affiliated with A2 (a large company), and contribute 33\% of all commits in Topcased (from January 2009, i.e. during the life span of both projects) and $21 \%$ of all commits in Papyrus. At affiliation level, A4 (an SME) is also active in both projects (as indicated in e.g. Figure 7), but with different committers in the two projects. The results indicate that there is considerable interaction between ecosystems, as a large proportion of the commits each month is provided by developers active in both projects. However, from our limited analysis of the time period for both projects it is difficult to establish any current and future trends in interactions between projects.

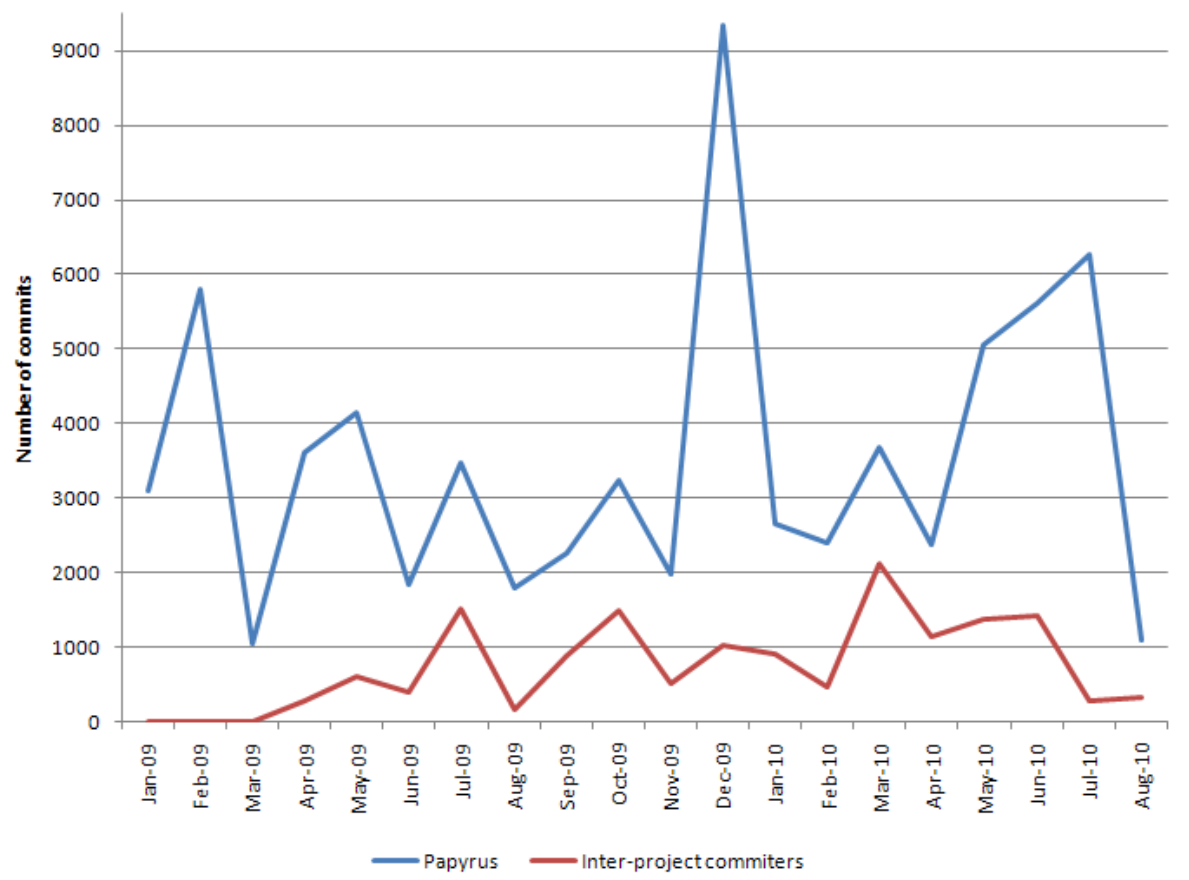

Fig. 14. Papyrus: total number of commits and contribution by inter-project committers

\section{Analysis}

There are a number of reasons for considering adoption of Open Source tools in a commercial context. For Combitech, development process optimisation based on MDD technology is an important service. This service is today mostly based on proprietary tools. Complementing this with Open Source tools would potentially have several benefits:

- Possibility to influence the development of the tool by contributing to the development. This could enable better support for the processes that Combitech is offering its customers to deploy. 
- Possibility to sell new services around the tool, for example custom made adaptations and bug fixing support.

- Today when offering tool experts, Combitech has a natural disadvantage towards the vendor of the tool which would not exist with an Open Source tool.

- The license cost for proprietary tools targeted towards the embedded market with full code generation capabilities is very high and can be a barrier hard to overcome for companies considering employing MDD. Being able to offer tooling with no license cost could be a door-opener to new customers.

- Eliminating the license cost needed for training courses in MDD technology. Today this cost makes it too expensive to offer these as open courses, they are only offered as customer specific courses held at customer sites with customer owned licenses.

- Combitech also works as a subcontractor developing software/hardware systems to customers. Eliminating license cost would make these services more competitive.

- The possibility to work in OSS development projects could be used to attract and keep developers to the company and act as an important part in their competence development.

In addition to these potential benefits from adoption of Open Source tools for MDD in the company context, it is also important for Combitech to consider the extent to which Topcased and Papyrus are likely to be sustainable and maintainable for a long time.

Considering the developer and user activity in the two projects, the co-occurence of peaks in the activity diagrams and events in the version history of the projects is not surprising, since it is expected that commits are performed in SCM repositories and that different matters are discussed in mailing lists in connection with new releases. However, this behaviour also indicates that there is a collaborative effort involved and that the community is responsive.

The activity in mailing lists and forums is rather limited for both investigated projects in contrast with other OSS projects like Nagios (IT infrastructure monitoring software) and the Apache webserver. For the corresponding user mailing lists in Nagios and Apache there are between 10 and 33 times as many messages each month according to Gamalielsson et al. (2010) and data from Gmane (http://gmane.org, accessed 7 January 2011). For the corresponding developer mailing lists there are between two and 19 times as many messages each month. However, Nagios and Apache, two projects aimed at the monitoring and operation of IT infrastructure, are more established projects with a broader user base. Another reason for the limited mailing list activity for the investigated tools may be extensive internal usage within organisations and also that other communication channels are used apart from public mailing lists and forums. Further, the maturity level of the projects may affect the activity in mailing lists.

Our analysis of the two OSS projects suggests that there is significant development involvement by companies and research institutes. This is in stark contrast with several other Open Source projects, where this kind of involvement is more limited, for example the projects Nagios, Mono and Evolution (Gamalielsson et al. 2010; 
Martinez-Romo et al. 2008). For the Topcased project there was a clear shift in affiliation amongst participants at a given point in time, where an SME that had been dominating since the start of the project was replaced by two dominating large companies. This shift of domination has also been observed in the Open Source project "Open XML/ODF translator" (Gamalielsson and Lundell 2010), where an SME was initially dominating but later replaced by another (somewhat larger) SME and a large company. A large company is backing up this project, and has also provided the specification for the tool. In the case of the investigated Papyrus project, committers affiliated with SMEs were also active early in the project, and large companies became more influential later on. The findings regarding contribution by affiliation may suggest that large companies engage in OSS projects with increased maturity and after initial releases of the tools.

From the results it is also evident that few committers contribute a large proportion of the commits. This is in agreement with the previous finding that "the vast majority of mature OSS programs are developed by a small number of individuals" (Krishnamurthy 2002), where it was found that the median number of developers was 4. As a comparison, the top five developers for Topcased and Papyrus contributed $84 \%$ and $78 \%$ of the commits, respectively. Further, Papyrus has more committers than the average incubator project on the Eclipse platform, as evidenced when comparing our results to a previous analysis of 23 incubator projects (Duenas et al. 2007).

There has been regular and frequent releases of the tools, and from the results we can not observe any decline in activity. On the contrary, it is likely that the interest and user base for the tools will increase in the future. One reason for this is that Papyrus is still in the "incubation phase" (Eclipse.org 2011b), but based on the current status of the project it is likely to be promoted to the mature phase soon, which in turn most likely will lead to an increased user base. It has also been reported that there is an on-going integration and consolidation effort of a number of Eclipse-based tools and technologies, which includes but is not limited to, Topcased and Papyrus (Eclipsecon.org 2010). An indication of this is our finding that several committers from the same large company contribute significantly to both projects. It is also a fact that an SME is active in both projects, but with different committers. The integration and consolidation effort could potentially lead to increased uptake within companies in the embedded systems domain.

Organisations that want to use/adopt OSS-based MDD tools need to engage in the ecosystems of the projects (Eclipse.org 2010). For our analysis of the two tools we found that both projects have a strong commercial involvement. Earlier experience (Lundell et al. 2010) from the Swedish context has identified a difference in the "collaboration climate between projects that have a strong commercial involvement and those that are more 'traditional', community-driven OS projects". Professional developers in Swedish companies developing with Open Source have also expressed concerns regarding a potential risk that a project is too strongly goal driven, leading to that "the fun and enthusiasm for those participating on a voluntary basis can disappear" (Lundell et al. 2010). In order to investigate the collaborative climate in each ecosystem, it would necessitate genuine interaction with each project in a pilot study. Without such it would be difficult to assess whether the collaborative climate 
in the Topcased and Papyrus ecosystems is congruent with existing work practices in the company.

In summary, our analysis suggests that both projects have healthy ecosystems, with an active base of developers and users. Despite a seemingly commercial drive, both projects seem to have developers representing an appropriate mix of different kinds of organisations involving the secondary software sector. This, in turn, can significantly contribute to long term sustainability for the OSS projects. For Combitech it is interesting to note that a number of practitioners representing large companies in the embedded systems domain have shown interest in Topcased, as evidenced by practitioner presentations at an industrial conference (Topcased.org 2011b). Further, in a consultancy report on the analysis of OSS-based MDD tools prepared on behalf of a large company in the embedded systems domain, a number of tools (including Topcased and Papyrus) were found promising and a more in-depth investigation of these was recommended (Eclipse.org 2010). In the same report it is also stated that of "particular interest and relevance is the TOPCASED project ...". In terms of functionality, the report also points out that "MDT Papyrus is still quite far from being ready for prime time, and would perhaps require additional attention and investment ..." (Eclipse.org 2010). This view is something which needs to be investigated in a pilot project within the organisation before adoption.

Our specific assessment of the health for two Open Source communities may have broader implications for evaluation and assessment of software systems. Evaluation of software systems has many dimensions and it is widely acknowledged to be a complex activity (Lundell and Lings 2004). Our specific strategy used for assessing health of Open Source ecosystems has certain similarities with, and may contribute to, previously proposed approaches (e.g. OpenBRR, QSOS, OMM) for evaluation and assessment of Open Source projects (Petrinja et al. 2010). Common to all these are that they are based on an a-priori evaluation framework (Lundell and Lings 2004). For example, QSOS features an intrinsic durability category which includes metrics such as activity on releases and number of developers. Similarly, OMM has number of commits and bug reports as metrics. Our activity analysis goes beyond previously presented approaches in that we elaborate on more intricate aspects such as affiliation and organisation types in our activity analysis. Further, a number of research projects (e.g. Flossmetrics and QualOSS) have systematically analysed OSS projects and as part of this proposed certain metrics (Daffara 2009).

\section{Conclusion and Future Work}

Prior to the initiation of this case study, the company explored the functionality offered in a number of Open Source MDD tools for the Eclipse platform for potential company adoption. The focus for this case study has been to explore the sustainability of the ecosystems for the two Open Source projects Topcased and Papyrus.

Overall, we found that both projects are active as evidenced by contributions in SCM repositories, mailing lists and forums. There seems to be an appropriate mix of professional organisations (SMEs, large companies and research institutes) involved in the development of the projects, and developers from one large company are active in both projects. From this we note that both Open Source projects have promising 
health in their respective ecosystems. Our analysis shows that it is likely that the external user base of the projects will increase over time as the projects mature and as a consequence of the on-going integration and consolidation effort of Eclipse-based tools and technologies. However, having studied two Open Source projects that have existed for less than a decade, only time can tell whether they are still active after several decades.

A limitation in our study concerning assessment of the user base is that we only consider publicly available project data in our analysis. From previous studies it is known that many large companies adopt and use Open Source software internally within their own organisational context. This is of particular importance in this domain with its very long life cycles. Further, we note that there is a lack of documented evidence that report on usage of OSS-based MDD tools in company contexts. One notable exception is the Topcased project for which it has been claimed that the Topcased tool "is in productive use in Airbus" (Eclipse.org 2009), and more specifically "Airbus Industry is committed to using TOPCASED in its A350 program" (Eclipse.org 2010). Further, the functionality and reliability of the Topcased tool has been found sufficient in other usage contexts where critical systems are being developed. For example, it has been used in the context of validation of satellite flight software (Pouly et al. 2011) and railway safety systems (Hase 2011). Motivated by such experiences from other companies in the secondary software sector and the results from our investigation of the Topcased community, the tool is currently being explored by developers at Combitech.

Based on our results, a natural next step for the company will be to proceed with a pilot study in order to analyse the extent to which the functionality offered in the investigated tools support the preferred working practice used in development projects in the company. Since the company perceives the health of the Topcased ecosystem to be promising and based on earlier positive experience of the tool, such a pilot study involving Topcased usage within the company context is seen as a natural next step. From this, the company will be able to further investigate the collaborative climate through genuine interaction between the company context and the broader Topcased ecosystem.

Acknowledgement. This research has been financially supported by the ITEA2 project OPEES (www.opees.org) through Vinnova (www.vinnova.se).

\section{References}

Bonaccorsi, A., Rossi, C.: Comparing motivations of individual programmers and firms to take part in the open source movement: from community to business. Knowledge Technology and Policy 18, 40-64 (2006)

Crowston, K., Howison, J.: The social structure of Free and Open Source software development. First Monday 10(2) (2005)

Crowston, K., Howison, J.: Assessing the Health of Open Source Communities. IEEE Computer 39(5), 89-91 (2006)

Daffara, C.: The SME guide to Open Source Software, 4th edn. FLOSSMETRICS report, European Commission project FP6-033982 (July 4, 2009), http: / / www. flossmetrics.org/sections/deliverables/docs /WP8/ D8.1.1-SMEs_Guide.pdf (accessed June 4, 2011) 
Duenas, J.C., Parada, G., H., A., Cuadrado, F., Santillan, M., Ruiz, J.L.: Apache and Eclipse: Comparing Open Source Project Incubators. IEEE Software 24(6), 90-98 (2007)

Ebert, C.: Open source software in industry. IEEE Software 25, 52-53 (2008)

Eclipse.org. Eclipse Automotive Interest Group- meeting minutes (2009), http: / / dev. eclipse.org/mhonarc/lists/auto-iwg/ pdfTcIV3Ghb68.pdf (accessed June 4, 2011)

Eclipse.org. Papyrus (2011a), http: / /www. eclipse.org/modeling/mdt/papyrus (accessed June 4, 2011)

Eclipse.org. Papyrus (2011b), http: / / www. eclipse.org/modeling/mdt (accessed June 4, 2011)

Eclipse.org. An Extended Survey of Open Source Model-Based Engineering Tools (2010), http://wiki.eclipse.org/images/d/dc/Report. external.bvs.pdf (accessed June 4, 2011)

Eclipsecon.org. Papyrus: Advent of an Open Source IME at Eclipse (2010), http: //www.eclipsecon.org/2010/sessions/sessions?id=1385 (accessed June 4, 2011)

Fitzgerald, B.: The Transformation of Open Source Software. MIS Quarterly 30(3), 587-598 (2006)

Gamalielsson, J., Lundell, B., Lings, B.: The Nagios community: An extended quantitative analysis. In: Ågerfalk, P., Boldyreff, C., González-Barahona, J.M., Madey, G.R., Noll, J., et al. (eds.) OSS 2010. IFIP AICT, vol. 319, pp. 85-96. Springer, Heidelberg (2010)

Gamalielsson, J., Lundell, B.: Open Source Software for Data Curation of Digital Assets: a case study. In: Lugmayr, A., et al. (eds.) Proceedings of Mindtrek 2010 of 14th International Digital Media \& Business Conference (MindTrek): Envisioning Future Media Environments, pp. 53-56. ACM, New York (2010)

German, D.: The GNOME project: a case study of open source global software development. Journal of Software Process: Improvement and Practice 8(4), 201-215 (2004)

Hase, K.R.: "openETCS": An Open Source Approach for Railway Safety Systems Adopting TOPCASED for CENELEC EN 50126/50128 Safety Case. In: First Topcased Days Toulouse 2011, Toulouse, France, February 2-4 (2011),

http: //gforge.enseeiht.fr/docman/view.php/52/4289/

A2-DeutscheBahn.pdf (accessed June 4, 2011)

Kamei, Y., Matsumoto, S., Maeshima, H., Onishi, Y., Ohira, M., Matsumoto, K.: Analysis of Coordination Between Developers and Users in the Apache Community. In: Russo, B., et al. (eds.) Open Source Development, Communities and Quality, pp. 81-92. Springer, Boston (2008)

Krishnamurthy, S.: Cave or Community? An Empirical Examination of 100 Mature Open Source Projects. First Monday 7(6) (2002)

van der Linden, F., Lundell, B., Marttiin, P.: Commodification of Industrial Software: A Case for Open Source. IEEE Software 26(4), 77-83 (2009)

Lopez-Fernandez, L., Robles, G., Gonzalez-Barahona, J.M., Herraiz, I.: Applying Social Network Analysis Techniques to Community-driven Libre Software Projects. International Journal of Information Technology and Web Engineering 1, 27-48 (2006)

Lundell, B., Bermejo, J., Labezin, C., Sempert, F., Valentin, M.-L., Laprevote, A., van der Linden, F., Pablos, J.J.: Open Source Software Workshop, ITEA 2 Symposium, Rotterdam, October 21 (2008)

Lundell, B., Lings, B.: On understanding evaluation of tool support for IS development. Australasian Journal of Information Systems (AJIS) 12(1), 39-53 (2004) 
Lundell, B., Lings, B., Lindqvist, E.: Open Source in Swedish companies: where are we? Information Systems Journal 20(6), 519-535 (2010)

Lundell, B., Lings, B., Syberfeldt, A.: Practitioner perceptions of Open Source software in the embedded systems area. Journal of Systems and Software (in press, 2011)

Martinez-Romo, J., Robles, G., Ortuño-Perez, M., Gonzalez-Barahona, J.M.: Using Social Network Analysis Techniques to Study Collaboration between a FLOSS Community and a Company. In: Russo, B., et al. (eds.) Open Source Development, Communities and Quality, pp. 171-186. Springer, Boston (2008)

Mattsson, A., Lundell, B., Lings, B., Fitzgerald, B.: Linking Model-Driven Development and Software Architecture: A Case Study. IEEE Transactions on Software Engineering 35(1), 83-93 (2009)

Mockus, A., Fielding, R.T., Herbsleb, J.D.: Two case studies of open source software development: Apache and Mozilla. ACM Transactions on Software Engineering and Methodology 11(3), 309-346 (2002)

Moon, Y.J., Sproull, L.: Essence of distributed work: The case of the Linux kernel. First Monday 5(11) (2000)

Ohloh.net. Topcased (2011a), http: / /www . ohloh. net/p/topcased (accessed June 4, 2011)

Ohloh.net. MDT Papyrus (2011b), http: / /www. ohloh. net/p/mdt-papyrus (accessed June 4, 2011)

Petrinja, E., Sillitti, A., Succi, G.: Comparing OpenBRR, QSOS and OMM Assessment Models. In: Ågerfalk, P., Boldyreff, C., González-Barahona, J.M., Madey, G.R., Noll, J., et al. (eds.) OSS 2010. IFIP AICT, vol. 319, pp. 224-238. Springer, Heidelberg (2010)

Pouly, J., Rolland, J.F., Faure, T., Hyounet, P., Zanon, O.: Automatic generation of tests from UML models to validate satellite flight software. In: First Topcased Days Toulouse 2011, Toulouse, France, February 2-4 (2011),

http: / / gforge. enseeiht. fr/docman/view.php/ 52 / (accessed June 4, 2011)

Robert, S.: New trends and needs for Avionics Systems. In: ARTEMIS Conference, Berlin (May 2007),

https: / /www.artemisia-association.org/downloads /

SYLVIE_ROBERT_AC_2007.pdf (accessed June 4, 2011)

Topcased.org. Topcased - The Open Source Toolkit for Critical Systems (2011a), http: / / www . topcased. org (accessed June 4, 2011)

Topcased.org. First Topcased Days Toulouse 2011, Toulouse, France, February 2-4 (2011b), http: / / www. topcased.org/index.php/content/view/53

(accessed June 4, 2011)

West, J.: How Open is Open Enough? Melding Proprietary and Open Source Platform Strategies. Research Policy 32(7), 1259-1285 (2003) 